L. A. Rubel

Nagoya Math. J.

Vol. 96 (1984), 23-28

\title{
A CHARACTERIZATION OF INTERNAL FUNCTIONS ON MULTIPLY CONNECTED REGIONS
}

\author{
LEE A. RUBEL
}

\section{Dedicated to the Memory of Errett Bishop}

The notion of internal function enters naturally in the study of factorization of function in Lumer's Hardy spaces-see [RUB], where this aspect is developed in some detail. By definition, a bounded analytic function $f$ on a complex-analytic manifold $M$ is internal if $\|f\|_{\infty}=1$ and if the following implication holds: if $h$ and $1 / h$ are bounded analytic functions on $M$ and if $|f| \leq|h| \leq 1$ everywhere on $M$, then $h$ must be a constant. It is shown in [RUB] that if $M=D$, the unit disc, then $f$ is internal if and only if $f$ is an inner function, i.e. $\left|f^{*}\right|=1$ a.e., where $f^{*}$ is the radial boundary function associated with $f$. In this paper, we take $M$ to be a region bounded by $n+1$ Jordan curves $\Gamma_{0}, \Gamma_{1}, \cdots, \Gamma_{n}$ and characterize the internal functions on $M$. This characterization (Theorem 1) is most useful in the case of an annulus $A$, in which case it has a simple expression. (See Corollary 2.) For domains of higher connectivity, it is not as easy to work with. However, it does follow (see Corollary 1) in the general case that if $f$ is internal then the boundary value function $f^{*}$ must have modulus 1 on at least one boundary component, a.e. with respect to harmonic measure $\omega$ on $\partial M$.

It would be interesting to similarly characterize Blaschke products on these regions $M$, but we cannot presently do so. (For $f$ to be a Blaschke product, one relaxes the condition on $h$ in the above definition of internal functions to the condition that $h$ and $1 / h$ are analytic (without demanding boundedness) on $M$.)

Our approach to the present characterization grew out of some valuable conversations we had some years back with Kenneth Stephenson, whom we thank for his help.

A major role is played here by the fluxes of a harmonic function,

Received May 31, 1983. 
so we now describe them. Throughout this paper, $M$ is a region bounded by $n+1$ closed Jordan curves $\Gamma_{0}, \Gamma_{1}, \cdots, \Gamma_{n}$, and $\Gamma_{0}$ is the outside boundary. Inside $M$, we draw $n+1$ analytic closed Jordan curves $\gamma_{0}, \gamma_{1}$, $\cdots, \gamma_{n}$ that run essentially parallel, respectively, to $\Gamma_{0}, \Gamma_{1}, \cdots, \Gamma_{n}$, but a little distance inside $M$, so that the $\gamma_{i}$ form a basis for the homology of $M$. If $w$ is a harmonic function on $M$, we define $F_{i}(w)$, the flux of $w$ around $\gamma_{i}$, by

$$
F_{i}=F_{i}(w)=\operatorname{Flux}_{r_{i}} w=\frac{1}{2 \pi} \int_{r_{i}} \frac{\partial w}{\partial \vec{n}} d s
$$

where $d s$ is arc length on $\gamma_{i}$ and $\partial / \partial \vec{n}$ denotes the (outward) normal derivative on $\gamma_{i}$-as usual, $\Gamma_{0}$ and $\gamma_{0}$ are oriented clockwise and the others counterclockwise. It is an elementary fact that

$$
F_{0}+F_{1}+\cdots+F_{n}=0 \text {. }
$$

Also, $e^{w}$ is the modulus of an analytic function on $M$ if and only if every $F_{i}$ is an integer [NEH]. This motivates our subsequent definition of $Z$ fluxable.

Throughout this paper, $f$ is an analytic function on $M$ with $\|f\|_{\infty}=$ $\sup \{|f(z)|: z \in M\}=1$, and we always take $u=-\log |f|$, so that $u$ is superharmonic on $M$. Now [FIS], $f$ has ("radial") boundary values $f^{*}$ defined a.e. with respect to harmonic measure on the boundary $\Gamma=\Gamma_{0}$ $+\Gamma_{1}+\cdots+\Gamma_{n}$ of $M$, and we let $u^{*}=-\log \left|f^{*}\right|$. Note that $u \geq 0$ and $u^{*} \geq 0$. We now let $\tilde{u}$ be the harmonic solution of the Dirichlet problem for the boundary values $u^{*}$ (see [FIS]). (Again, $\tilde{u} \geq 0$ on $M$.)

Definition. $f$ is $Z$-fluxable if $\tilde{u}$ has a non-negative harmonic minorant $\tilde{v}$ which is not identically 0 , with every flux of $\tilde{v}$ an integer, i.e. $F_{i}(\tilde{v}) \in Z, i=0,1, \cdots, n$.

THEOREM 1. The non-constant bounded analytic function $f$ on the multiply connected Jordan region $M$, with $\|f\|_{\infty}=1$, is internal if and only if $f$ is not Z-fluxable.

Proof. (Easy) Suppose $f$ is internal, but is $Z$-fluxable. Take the function $\tilde{v}$ in the definition of $Z$-fluxability, and let $\exp -\tilde{v}=|h|$. This $h$ violates the definition of $f$ being internal, so we have proved by contradiction that internal $\Rightarrow$ not $Z$-fluxable. Similarly suppose $f$ is not $Z$ fluxable but not internal. Then take the function $h$ that makes $f$ not internal, and let $\tilde{v}=-\log |h|$, etc. 
Corollary 1. If $f$ is internal on such an $M$, then $\left|f^{*}\right|=1$ a.e. (with respect to harmonic measure $\omega$ ) on at least one boundary component $\Gamma_{i}$ of $\Gamma$.

Proof. Suppose on the contrary, that each $\Gamma_{i}$ has a set $E_{i}$ of positive harmonic measure, such that $\left|f^{*}\right|<1-\varepsilon$ on every $E_{i}, i=0,1, \cdots, n$, i.e. $u \geq \log 1 /(1-\varepsilon)=\delta$ say. Take $v$ as the solution of the Dirichlet problem, with boundary values 0 on $\Gamma \backslash\left(\cup E_{i}\right)$ and boundary values $\delta_{i}$ on $E_{i}, i=$ $0,1, \cdots, n$ where $0 \leq \delta_{i} \leq \delta$ are at our disposal. Clearly, $0 \leq \tilde{v} \leq \tilde{u}$, and $\tilde{v}$ is not identically zero. Let us normalize the $\delta_{i}$ so that $\delta_{0}+\delta_{1}+\cdots+\delta_{n}$ $=\delta$. Then the point $\left(\delta_{0}, \delta_{1}, \cdots, \delta_{n}\right)=\tilde{\delta}$ lies on a standard $n$-simplex $\Sigma$ in $n$-space. For ease of exposition, we shall take $n=2$, although the corresponding arguments work for larger $n$. By considerations of heat flow, we have

$$
\left\{\begin{array}{l}
F_{0}\left(0, \delta_{1}, \delta_{2}\right)<0 \\
F_{1}\left(\delta_{0}, 0, \delta_{2}\right)<0 \\
F_{2}\left(\delta_{0}, \delta_{1}, 0\right)<0 .
\end{array}\right.
$$

Let $F$ be the vector $F=\left\langle F_{0}, F_{1}\right\rangle$ in $R^{2}$. Label the vertices of $\Sigma$ as $A, B, C$, where $A=(0, \delta, 0), B=(0,0, \delta)$ and $C=(\delta, 0,0)$. We shall prove that there is a point $\sigma \in \Sigma$ where $F=0$, so that $f$ will actually be $Z$ fluxable contrary to Theorem 1. At this point, our proof becomes topological, and is similar to proofs of the Brouwer fix-point theorem. [We thank Richard Jerrard for his generous help with the topological part of the proof.] Geometrically, our conditions $(\alpha)$ say that $F$ maps $\overline{A B}$ into the open left half-plane $x<0, F$ maps $\overline{C A}$ into the open lower half-plane $y<0$, and $F$ maps $\overline{B C}$ into the open upper-right half-plane $x+y>0$. If $A^{\prime}=F(A), B^{\prime}=F(B)$ and $C^{\prime}=F(C)$, then $A^{\prime}$ lies in the quadrant $x<0, y<0, B^{\prime}$ lies in the wedge $x<0, x+y>0$, and $C^{\prime}$ lies in the wedge $y<0, x+y>0$. It is now apparent that the index (winding number) of $F(\partial \Sigma)$ is exactly 1, and it follows from a simple homotopy argument that $F$ must have at least one zero in $\Sigma$. The same proof works for higher $n$, but is more complicated to express. (See [DUG], for example, for a definition of the index in higher dimensions.) We omit the details of the proof. Since the "intermediate-value theorem" we are establishing here might be of independent interest, we state it separately.

Theorem 2. Let $\Sigma$ be the standard $n$-simplex in $\boldsymbol{R}^{n+1}$ given by $x_{0}+$ $x_{1}+\cdots+x_{n}=1$. Suppose $F=\left\langle F_{0}, F_{1}, \cdots, F_{n}\right\rangle$ maps $\Sigma$ into $R^{n+1}$ and satisfies $F_{0}+F_{1}+\cdots+F_{n}=0$. Suppose further that 


$$
\left\{\begin{array}{c}
F_{0}\left(0, x_{1}, x_{2}, \cdots, x_{n}\right)<0 \\
F_{1}\left(x_{0}, 0, x_{2}, \cdots, x_{n}\right)<0 \\
\vdots \\
F_{n}\left(x_{0}, x_{1}, \cdots, x_{n-1}, 0\right)<0 .
\end{array}\right.
$$

Then there is a point $\sigma \in \Sigma$ with $F(\sigma)=0$.

Remark. Dmitri Khavinson has written the author that Corollary 1 of this paper can alternatively be proved by using Lemma 2 of his paper [KHA II], which involves only linear algebra, without topology.

Corollary 2. In the annulus $A=A_{r}=\{z \in C: 1<|z|<r\}$, a bounded non-constant analytic function $f$ with $\|f\|_{\infty}=1$ is internal iff both

i) $\left|f^{*}\right|=1$ a.e. on at least one boundary component of $A$ and

ii) $\mid$ Flux $\tilde{u} \mid<1$.

(Here $\tilde{u}$ is as described above, namely the solution to the Dirichlet problem with boundary values $-\log \left|f^{*}\right|$.)

The proof is along the lines of what we have already done, but is much simpler, so we omit it. For $n \geq 2$, the condition of $Z$-fluxability seems to be much more delicate than in the annulus case $n=1$, and probably depends in subtle ways on the detailed geometry of the region as well as the boundary values of $f$.

Let us now give an application of Theorem 1 that we see no other way of proving

COROLlaRy 3. Let $g$ be a bounded analytic function on the multiplyconnected Jordan region $M$ as above, and let $m_{g}$ be the operator on $H^{\infty}(M)$ of multiplication by $g$, i.e. $m_{g}(f)=g f$. Then $m_{g}(f)$ is internal for every internal function $f$ if and only if $m_{g}$ is an (into) isometry of $H^{\infty}(M)$.

Proof. It is proved in [HAR] that $m_{g}$ is an isometry if and only if $|g| \equiv 1$ on $S$, the Shilov boundary of $H^{\infty}(M)$, and that this happens if and only if $\left|g^{*}\right|=1$ almost everywhere on $\partial M$. Since $f$ being not $Z$-fluxable (equivalently internal) depends only on $\left|f^{*}\right|$ it is clear that if $\left|g^{*}\right|=1$ a.e. then $f$ and $g f$ are internal together. We omit the details of the proof of the converse implication, which follow along the lines of the rest of this paper.

It would be interesting to know whether, if $\varphi$ is an arbitrary linear isometry of $H^{\infty}(M)$ into $H^{\infty}(M)$, then $\varphi(f)$ must be internal if $f$ is internal. 
If $\varphi$ is onto, this follows directly from the definition of internal and the characterization (see [FIS]) of onto linear isometries $\varphi$ as being of the form $\varphi(f)=\lambda[f \circ a]$, where $\lambda$ is a constant of modulus 1 and $a$ is an analytic map of $M$ onto $M$. No-one seems to know the general linear isometry of $H^{\infty}(M)$ into $H^{\infty}(M)$, but it is tempting to guess that it has the form $\varphi(f)=g \times[f \circ a]$, where $g$ is as above, and $a$ maps $M$ into $M$ so that the range of a contains a dominating set for $H^{\infty}(M)$.

We end with a word about our References section, which is unusually long, and is intended to be so. There is a great deal of Soviet literature of function spaces on multiply connected domains that seems to have escaped the attention of Western authors, and we feel that a real purpose will be served by giving a more extensive bibliography than is usual.

\section{REFERENCES}

[ABR] M. B. Abrahamse, The Pick interpolation theorem for finitely connected domains, Michigan Math. J., 26 (1979), 195-203.

[ALS] I. A. Alexandrov and A. S. Sorokin, The Schwarz problem in multiplyconnected circular domains (in Russian), Siberian Math. J., XIII No. 5 (1972), 971-1001.

[COW] R. Coifman and G. Weiss, A kernel associated with certain multiply connected domains and its applications to factorization theorems, Studia Math., XXVIII (1966), 31-68.

[DUG] James Dugundji, Topology, Allyn and Bacon, 1966.

[DUK I] L. E. Dunduchenko and S. A. Kasyanuk, Bounded analytic functions in multiply connected domains (in Ukrainian), Dokl. Akad. Nauk Ukrain. SSR, No. 2 (1959), 111-115.

[DUK II] — On subclasses of analytic functions of the Nevanlinna class in $n$-connected circular domains (in Ukrainian) Dokl. Akad. Nauk, Ukrain. SSR, No. 9 (1959), 994-948.

[DUK III] —, Functions of bounded type in a circular annulus, Acad. R. P. Romine An. Romino-Soviet Ser. Mat.-Fiz (3) (1962) no. 2 (41) 63-81.

[DUN] L. E. Dunduchenko, On the changing of certain analytic functions so that they become single-valued in multiply-connected domains (In Russian) Math. USSR-Sb., 67 (109): 1 (1965), 3-15.

[FIS] Stephen D. Fisher, Function Theory on Planar Domains, John Wiley and Sons, 1983.

[HAR] Morisuke Hasumi and Lee A. Rubel, Multiplication isometries of Hardy spaces and of double Hardy spaces, Hokkaido Math. J., 10 (1981), 221-241.

[HAS I] M. Hasumi, Invariant subspace theorems for finite Riemann surfaces, Canad. J. Math., 18 (1966), 240-255.

[HAS II] - Hardy classes on plane domains, Ark. Mat., 16 (1978), 213-227.

[HAT I] S. Ya Havinson and G. C. Tumarkin, The existence of a single-valued analytic function of a given class with a given modulus of boundary values in multiply-connected domains (in Russian) Izv. Akad. Nauk. SSSR, Ser. Mat., 22, No. 4, pp. 3-52.

[HAT II] S. Ya Havinson and G. C. Tumarkin, The possibility of representing a 
harmonic function by the Green formula in a multiply-connected domain (in Russian), Math. USSR-Sb., 44 (86): 2 (1958), 225-234.

[HAT III] S. Ya Havinson and G. C. Tumarkin, Classes of analytic functions in multiply-connected domains, "Investigations in modern problems of the theory of complex-variable functions," edited by A. I. Markushevich (in Russian), Moscow 1960.

[HAV] S. Ya Havinson, Removable sets of analytic functions in the Smirnov class, sb. "Certain problems in the modern theory of functions" (in Russian), Akad. Nauk SSSR, Sib. ser. Mathem. (1976), 160-166.

[HEI] M. Heins, Hardy Classes on Riemann Surfaces, Lecture Notes in Mathematics, 98 (1969), Springer-Verlag, Berlin, Heidelberg and New York.

[HEJ] D. Hejhal, Classification theory for Hardy classes of analytic functions, Ann. Acad. Sci. Fenn, Ser. AI, 566, Helsinki 1973.

[KAS] S. A. Kasyanuk, On functions of class $A$ and $H_{g}$ on an annulus, Math. USSR-Sb. (Mat. Sb.), 42 (84), (1967), 301-326.

[KHA I] D. S. Khavinson, Factorization theorems for different classes of analytic functions in multiply-connected domains, Preprint 1981.

[KHA II] D. S. Khavinson, On removal of periods of conjugate functions in multiply connected domains, Preprint 1981.

[KUZ] T. S. Kuzina, Parametrization in certain classes of analytic functions in multiply-connected domains (in Russian) Izv. Vuzov, ser. Mathem. (to appear).

[NEH] Zeev Nehari, Conformal Mapping, McGraw-Hill, 1952.

[NEV] C. W. Neville, Invariant Subspaces of Hardy Classes on Infinitely Connected Open Surfaces, Mem. Amer. Math. Soc., 2 (1975), Number 160.

[ROB] R. M. Robinson, Analytic functions in circular rings, Duke J. Math., 10 (1943), 341-354.

[RUB] Lee A. Rubel, Internal-external factorization in Lumer's Hardy spaces, Adv. in Math., to appear.

[SAR] D. E. Sarason, The $H p$ spaces of an annulus, Mem. Amer. Math. Soc., 56 (1965), 1-78.

[TAM] P.M. Tamrazov, The generalization of the Blaschke product in multiplyconnected domains (in Ukrainian), Dokl. Akad. Nauk. Ukrain. SSR, No. 7 (1962), 853-856.

[VOI] M. Voichik, Ideals and invariant subspaces of analytic functions, Trans. Amer. Math. Soc., 111 (1964), 493-512.

[VOZ] M. Voichik and L. Zalcman, Inner and outer functions on Riemann surfaces, Proc. Amer. Math. Soc., 16 (1965), 1200-1204.

[ZAL] L. Zalcman, Bounded analytic functions on domains of infinite connectivity, Trans. Amer. Math. Soc., 144 (1969), 241-269.

[ZMO] V. A. Zmorovich, The generalization of the Schwarz formula for multiplyconnected domains (in Ukrainian), Dokl. Akad. Nauk. Ukrain. SSR, No. 4 (1962), 853-856.

[ZVE] E. I. Zverovich, Boundary problems in the theory of analytic functions in the Hölder classes on Riemann surfaces (in Russian) Uspehy. Mathem. Nauk. 26, No. 1, 1971, 113-179. 